\title{
Stationarity in moment closure and quasi-stationarity of the SIS model
}

\author{
José Martins ${ }^{\mathrm{a}, \mathrm{b}, *}$, Alberto Pinto ${ }^{\mathrm{b}, \mathrm{c}}$, Nico Stollenwerk ${ }^{\mathrm{d}}$ \\ ${ }^{a}$ Department of Mathematics, School of Technology and Management, Polytechnic Institute of Leiria, Campus 2, Morro do Lena - Alto do Vieiro, 2411-901 Leiria, Portugal \\ ${ }^{\mathrm{b}}$ LIAAD-INESC Porto LA, Portugal \\ ${ }^{\mathrm{c}}$ Department of Mathematics, Faculty of Sciences, University of Porto, Rua do Campo Alegre, 687, 4169-007 Porto, Portugal \\ ${ }^{\mathrm{d}}$ CMAF, Faculdade de Ciências da Universidade de Lisboa, Av. Prof. Gama Pinto 2, 1649-003 Lisboa, Portugal
}

\section{A R T I C L E I N F O}

\section{Article history:}

Received 26 April 2011

Received in revised form 24 October 2011

Accepted 14 February 2012

Available online 23 February 2012

\section{Keywords:}

SIS model

Moment closure

Quasi-stationary states

Moments dynamic

\begin{abstract}
A B S T R A C T
Previous epidemiological studies on SIS model have only considered the dynamic evolution of the mean value and the variance of the infected individuals. In this paper, through cumulant neglection, we use the dynamic equations of all the moments of infected individuals to develop a recursive method to compute the equilibria manifold of the moment closure ODE's. Specifically, we use the stable equilibria of the moment closure ODE's to obtain good approximations of the quasi-stationary states of the SIS model. This is a crucial step when the quasi-stationary distribution is highly skewed.
\end{abstract}

(c) 2012 Elsevier Inc. All rights reserved.

\section{Introduction}

The stochastic SIS model, introduced by Weiss and Dishon [24], is used to study endemic diseases that do not confer immunity, such as malaria (see Bailey [1], Brauer and Castillo-Chavez [3], Brauer et al. [4], Renshaw [20]). Other practical applications include well-known diseases such as tuberculosis and meningitis (see Hethcote [6]). Typically these diseases are transmitted by bacterial and protozoan agents. The stochastic SIS model is equivalent to the well-known stochastic logistic model in population dynamics (see Nåsell [13]). In this paper we focus mainly on how epidemics spread in small environments, like schools, and on population dynamics of small neighborhoods.

The stochastic SIS model is a birth-and-death process with a finite state space corresponding to the number of infected individuals $I(t)$, at time $t$, and susceptible individuals $S(t)=N-I(t)$, where $N$ is the total number of individuals. Since the state $I(t)=0$ is the only absorbing state, the stationary distribution is degenerated with a probability of one at this state. Since the time to reach the equilibrium $I(t)=0$ can be quite long, the stationary distribution is non-informative and therefore it is critical to compute the quasi-stationary distribution, i.e. the stationary distribution of the stochastic process conditioned to the non-extinction of the infected

* Corresponding author at: Department of Mathematics, School of Technology and Management, Polytechnic Institute of Leiria, Campus 2, Morro do Lena - Alto do Vieiro, 2411-901 Leiria, Portugal. Tel.: +351 244820 300; fax: +351 244820310.

E-mail addresses: jmmartins@ipleiria.pt (J. Martins), aapinto1@gmail.com (A. Pinto), nico@ptmat.fc.ul.pt (N. Stollenwerk). individuals (see Kryscio and Lefevre [7], Nåsell [12]). The quasistationary distribution does not have an explicit form because when it is applied the moment generating function to both sides of the Kolmogorov forward differential equation of the SIS process, the differential equation with respect to the $m$ moment of infected individuals depends on the $(m+1)$ moment. Moment closure methods can be applied to do the replacement of the $(m+1)$ moment in the differential equation of the $m$ moment by an expression containing only lower-order moments. Usually moment closure methods are based on the assumption of specific distribution families or on cumulant truncation (see Matis and Guardiola [10], Matis and Kiffe [11], Singh and Hespanha [21]), although there are no specific distribution families beyond the normal distribution such that all high cumulants are zero (see Lukacs [9]). Hence, each moment closure method determines a way to close the dynamics of the moments, which leads to different results.

Nåsell [15] studied the first 3 cumulants for the stochastic SIS model. In the cases where the basic reproduction ratio is distinctly larger than 1 , and the population size $N$ is large, the closure method used consisted in setting the third cumulant equal to zero based upon the assumption that the distribution is asymptotically normal. In the cases where the distributions are skewed, the closure method used consisted in using cumulant truncation by setting the fourth cumulant equal to zero. Krishnarajah et al. [8] explored the use of mixture distributions, based on a mixture of a point mass at zero representing extinction of the process, and log-normal and beta-binomial distributions to approximate the distribution conditional upon non-extinction. Clancy and Mendy [5] found that: (i) in the subcritical region, a geometric distribution approximation 
to the quasi-stationary distribution of an SIS model is preferred; (ii) in the supercritical region, a beta-binomial distribution is preferred. Here, we attempt to improve the approximations by truncating at higher order cumulants. To accomplish this we developed a recursive method to compute the equilibria manifold of the $m$ moment closure ODE's, for every $m$. We show that for high values of $m$ this method provides better approximations when the quasi-stationary distribution is highly skewed, which is more likely the case when either the population size is small or the infection rate is small or both.

\section{The stochastic SIS model}

We consider the stochastic SIS (Susceptible-Infected-Susceptible) model that describes the evolution of an infectious disease in a population of $N$ individuals, where each individual can be either infected or susceptible. Denoting the global quantity of susceptible individuals by $S(t)$ and the infected quantity by $I(t)$, at time $t$, we obtain that $S(t)+I(t)=N$. The stochastic SIS model is a birth-and-death process with a finite state space, correspondent to the number of infected individuals $I(t) \in\{0,1,2, \ldots, N\}$ at time $t$. The birth rate $\beta$ is the probability, per unit of time, of one infected individual to have a contact with a susceptible one and to transmit the disease. The death rate $\alpha$ is the probability, per unit of time, of one infected individual to recover and become susceptible again. In an epidemiological context, the rate $\beta$ is known as the infection rate and $\alpha$ as the recovery rate. Thus, in the SIS model the spreading of the epidemic can be illustrated by

$S+I \stackrel{\beta}{\rightarrow} I+I \quad$ and $\quad \stackrel{\alpha}{\rightarrow} S$.

Let $p(I, t)$ denotes the probability of having $I$ infecteds at time $t$. The time evolution of the $p(I, t)$ is given by the master equation of the SIS model

$$
\begin{aligned}
\frac{d}{d t} p(I, t)= & \beta \frac{N-(I-1)}{N}(I-1) p(I-1, t)+\alpha(I+1) p(I+1, t) \\
& -\left(\beta \frac{N-I}{N} I+\alpha I\right) p(I, t)
\end{aligned}
$$

with $I \in\{0,1, \ldots, N\}$. The master equation is a gain-loss term equation, also known as the differential form of the Chapman-Kolmogorov equation, described for general Markov processes in Bailey [2] and especially applied to chemical reactions in van Kampen [23].

\subsection{Quasi-stationary distribution}

The state $I(t)=0$ is the only one absorbing and is attained for a finite time, i.e., the stationary distribution of the stochastic SIS model is degenerated with probability one at the origin

$$
p\left(I^{*}=k\right)= \begin{cases}1, & \text { if } \quad k=0, \\ 0, & \text { if } \quad k \neq 0,\end{cases}
$$

However, the time to reach the equilibrium $I(t)=0$ can be so long that the stationary distribution is non informative and the interest goes to the quasi-stationary distribution. The quasi-stationary distribution is the stationary distribution of the same stochastic process conditioned to the non-extinction of the infected individuals

$\{I(t)=i \mid I(t)>0\}, \quad i=1,2, \ldots, N$,

and therefore supported on the set of the transient states. Denoting by $\tilde{q}_{i}(t)$ the probability of having $i$ infecteds in the conditioned process, at time $t$, we obtain

$\tilde{q}_{i}(t)=p(I(t)=i \mid I(t)>0)=\frac{p(I(t)=i)}{1-p(I(t)=0)}, \quad i=1,2, \ldots, N$

Hence, the quasi-stationary distribution is the solution of the equation $\frac{d}{d t} \tilde{q}_{i}(t)=0$. Let $q_{i}$ denote these solution probabilities. Observing that $\frac{d}{d t} p(I(t)=0)=\alpha p(I(t)=1)$ and applying the master equation we obtain that

$\lambda_{i-1} q_{i-1}-k_{i} q_{i}+\mu_{i+1} q_{i+1}=-\alpha q_{1} q_{i}$,

where

$\lambda_{i-1}=\beta \frac{N-(i-1)}{N}(i-1), \quad k_{i}=\beta \frac{N-i}{N} i+\alpha i$

and $\mu_{i+1}=\alpha(i+1)$

We observe that the system Eq. (2) can not be solved explicitly because of the product $q_{1} q_{i}$ in the right-hand side of Eq. (2), but we can put all $q_{i}$ probabilities depending on $q_{1}$. Hence, $q_{i}$ satisfies the relation

$q_{i}=\gamma(i) \alpha(i) R_{0}^{i-1} q_{1}, \quad i=1,2, \ldots, N$,

where $R_{0}=\beta / \alpha$,

$\gamma(i)=\frac{1}{i} \sum_{k=1}^{i} \frac{1-\sum_{l=1}^{k-1} q_{l}}{\alpha(k) R_{0}^{k-1}}$,

$\alpha(i)=\frac{N !}{(N-i) ! N^{i}}$,

and

$q_{1}=\frac{1}{\sum_{i=1}^{N} \gamma(i) \alpha(i) R_{0}^{i-1}}$.

Eqs. (3)-(6) do not define explicitly the quasi-stationary distribution. However, we can use iterative methods to approach the $q_{i}$ values (see Nåsell [14]). One possible method starts with an initial guess for $q_{1}$ and uses Eq. (3) to determine the other $q_{i}$. Then $q_{1}$ can be actualized using Eq. (6). This process should be repeated until successive iterations are close enough. This iterative method converges because the quasi-stationary distribution $q_{i}$ is a left eigenvector of a matrix $A_{Q}$ (see Nåsell [12]), that has real and distinct negative eigenvalues (see Picard [17]), and the correspondent eigenvalue is the dominant one.

\subsection{Approximating the quasi-stationary distribution}

Since the quasi-stationary distribution of the stochastic SIS model does not have an explicit form, it is useful to approximate the model in order to obtain explicit approximations of the quasi-stationary distribution. Two possible approximations were studied by Kryscio and Lefevre[7] and by Nåsell [12,13]. One is given by the stationary distribution of the SIS model with one permanently infected individual and the other is obtained from the SIS model with the state $I(t)=0$ removed, i.e., the recovery rate is zero when there exists only one infected individual while all the other transition rates stay unchanged. The stationary distribution of this last process can be determined explicitly and gives

$p_{j}=\frac{N !}{j(N-j) ! N^{j}}\left(\frac{\beta}{\alpha}\right)^{j-1} p^{(0)}, \quad j=1,2, \ldots, N$,

where $p_{j}=P\left(I^{*}=j\right)$ and $p^{(0)}$ is defined by

$p^{(0)}=\frac{1}{\sum_{j=1}^{N} \frac{N !}{j(N-j) ! N^{j}}\left(\frac{\beta}{\alpha}\right)^{j-1}}$.

This stationary distribution defines a good approximation of the quasi-stationary distribution of the SIS model when the basic reproduction ratio is distinctly larger than 1 , i.e., the infection rate $\beta$ is distinctly greater than the recovery rate $\alpha$, and $N \rightarrow+\infty$ (see Nåsell [13]). In Fig. 1, we compare the mean value of infecteds that arises from the quasi-stationary distribution $\langle I\rangle_{Q S}$ with the mean value $\langle I\rangle_{Q S, A p x}$ of the approximated distribution presented in Eq. (7). The 

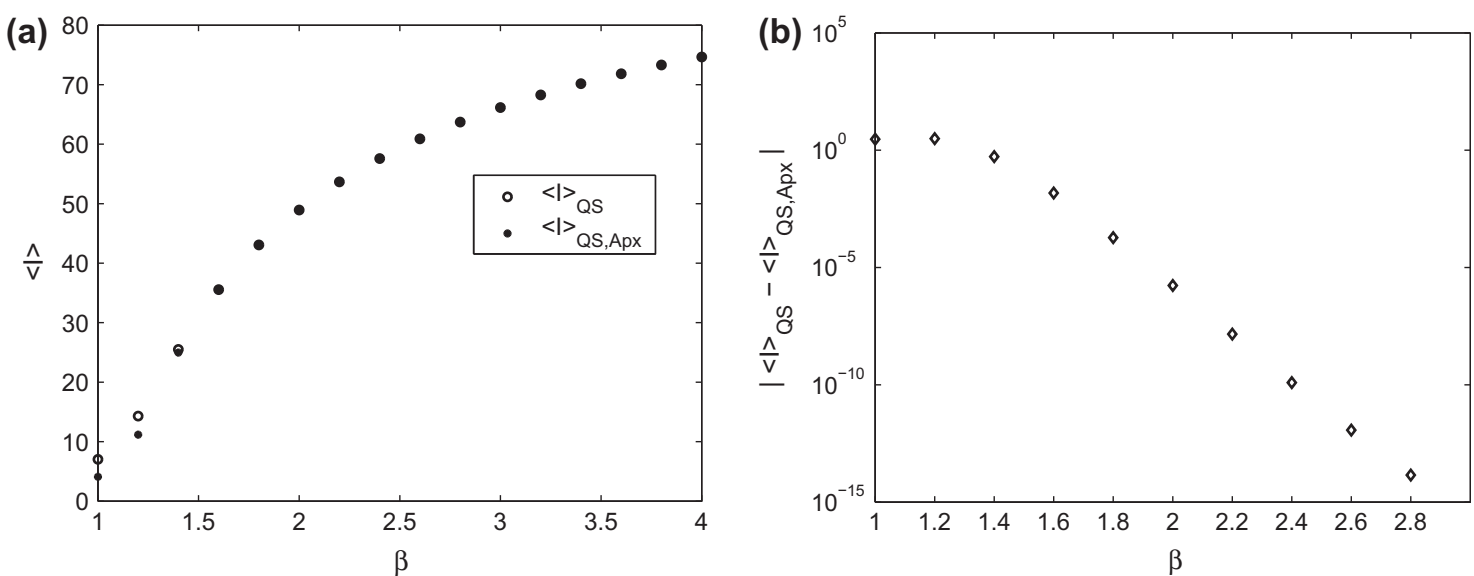

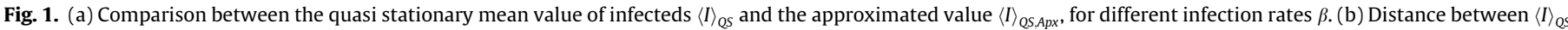
and $\langle I\rangle_{Q S, A p x}$. Parameters used: $N=100$ and $\alpha=1$.

quasi-stationary mean value was computed iteratively from Eqs. (3) to (6) with a precision of 15 digits. The parameters used are $N=100$ and $\alpha=1$.

\section{The $m$ moment closure SIS ODE's}

We will derive the dynamics of the $m$ first moments of infecteds $\langle I\rangle,\left\langle I^{2}\right\rangle, \ldots,\left\langle I^{m}\right\rangle$, for the $m$ moment closure ODE's through cumulant truncation and we will compute the stable equilibria manifold. We will study when the stable equilibria $\langle I\rangle_{m, \beta}^{*}$ can be a good approximation of the quasi-stationary mean value of infecteds $\langle I\rangle_{Q S, \beta}$.

\subsection{Moment closure}

Let $\left\langle I^{n}\right\rangle$ denote the $n t h$ moment of the state variable $I$ that is given by

$\left\langle I^{n}\right\rangle(t)=\sum_{I=0}^{N} I^{n} p(I, t)$.

The ODE of the nth moment of infecteds, derived from the master equation, (see Krishnarajah et al.[8]) is given by

$\frac{d}{d t}\left\langle I^{n}\right\rangle=f_{n}\left(\langle I\rangle,\left\langle I^{2}\right\rangle, \ldots,\left\langle I^{n}\right\rangle\right)-\frac{\beta}{N} n\left\langle I^{n+1}\right\rangle$,

where

$$
\begin{aligned}
f_{n}\left(\langle I\rangle,\left\langle I^{2}\right\rangle, \ldots,\left\langle I^{n}\right\rangle\right)= & \sum_{j=1}^{n}\left(\begin{array}{c}
n \\
j
\end{array}\right)\left(\beta+(-1)^{j} \alpha\right)\left\langle I^{n+1-j}\right\rangle \\
& -\frac{\beta}{N} \sum_{j=2}^{n}\left(\begin{array}{c}
n \\
j
\end{array}\right)\left\langle I^{n+2-j}\right\rangle .
\end{aligned}
$$

The characteristic function $p: \mathbb{R} \rightarrow \mathbb{C}$ of the state variable $I$ is defined by

$p(k)=\left\langle e^{i k I}\right\rangle=\sum_{I=0}^{N} e^{i k I} p(I, t)$,

and the $n t h$ cumulant of $I$ is the constant $k_{n}$ defined implicitly by

$\ln p(k)=\sum_{n=1}^{\infty} k_{n} \frac{(i k)^{n}}{n !}$.

We denote the $n$th cumulant of $I$ by $k_{n}=\left\langle\left\langle I^{n}\right\rangle\right\rangle$. There is a relation between cumulants and moments (see Smith [22], Papoulis [16]) given by $\left\langle\left\langle I^{n+1}\right\rangle\right\rangle=\left\langle I^{n+1}\right\rangle-\sum_{j=1}^{n}\left({ }^{n}{ }_{j}\right)\left\langle I^{j}\right\rangle,\left\langle\left\langle I^{n+1-j}\right\rangle\right\rangle, \quad n \geqslant 1$,

where the first cumulant is equal to the mean value of the infecteds $\langle\langle I\rangle\rangle=\langle I\rangle$. To apply the cumulant truncation technique, we will use the following Lemma that is proved in A.

Lemma 1. Assuming that $\left\langle\left\langle I^{m+1}\right\rangle\right\rangle=0$, there is a polynomial function $g_{m}\left(\langle I\rangle,\left\langle I^{2}\right\rangle, \ldots,\left\langle I^{m-1}\right\rangle\right)$ such that

$$
\left\langle I^{m+1}\right\rangle=g_{m}\left(\langle I\rangle,\left\langle I^{2}\right\rangle, \ldots,\left\langle I^{m-1}\right\rangle\right)+(m+1)\langle I\rangle\left\langle I^{m}\right\rangle .
$$

The moment closure technique was introduced by Whittle [25]. The mth moment closure approximation consists in assuming that the state variables are normally distributed, hence $\left\langle\left\langle I^{m+1}\right\rangle\right\rangle=0$ for $m \geqslant 2$, and therefore in replacing the moment $\left\langle I^{m+1}\right\rangle$ in Eq. (10) by the expression given in Eq. (15). This moment closure technique is known by cumulant truncation or cumulant negletion. Hence, the $m$ moment closure ODE's for the $m$ first moments of infecteds $\langle I\rangle,\left\langle I^{2}\right\rangle, \ldots,\left\langle I^{m}\right\rangle$, after applying the cumulant truncation technique, is as follows:

- for $n=1, \ldots, m-1$, the ODE's of $\left\langle I^{n}\right\rangle$ are as presented in Eq. (10);

- the ODE of $\left\langle I^{m}\right\rangle$ is given by

$$
\begin{aligned}
\frac{d}{d t}\left\langle I^{m}\right\rangle= & f_{m}\left(\langle I\rangle,\left\langle I^{2}\right\rangle, \ldots,\left\langle I^{m}\right\rangle\right) \\
& -\frac{\beta}{N} m\left[g_{m}\left(\langle I\rangle,\left\langle I^{2}\right\rangle, \ldots,\left\langle I^{m-1}\right\rangle\right)+(m+1)\langle I\rangle\left\langle I^{m}\right\rangle\right]
\end{aligned}
$$

where $f_{m}$ and $g_{m}$ are as presented in Eq. (11) and Lemma 1, respectively.

The resulting $m$ moment closure ODE's are therefore given by

$\frac{d}{d t}\langle I\rangle=(\beta-\alpha)\langle I\rangle-\frac{\beta\left\langle I^{2}\right\rangle}{N}$

$\frac{d}{d t}\left\langle I^{2}\right\rangle=(\beta+\alpha)\langle I\rangle+2(\beta-\alpha)\left\langle I^{2}\right\rangle-\frac{\beta}{N}\left\langle I^{2}\right\rangle-2 \frac{\beta}{N}\left\langle I^{3}\right\rangle$

$\frac{d}{d t}\left\langle I^{m}\right\rangle=f_{m}\left(\langle I\rangle,\left\langle I^{2}\right\rangle, \ldots,\left\langle I^{m}\right\rangle\right)-\frac{\beta}{N} n\left[g_{m}\left(\langle I\rangle,\left\langle I^{2}\right\rangle, \ldots,\left\langle I^{m-1}\right\rangle\right)\right.$

$\left.+(m+1)\langle I\rangle\left\langle I^{m}\right\rangle\right]$. 

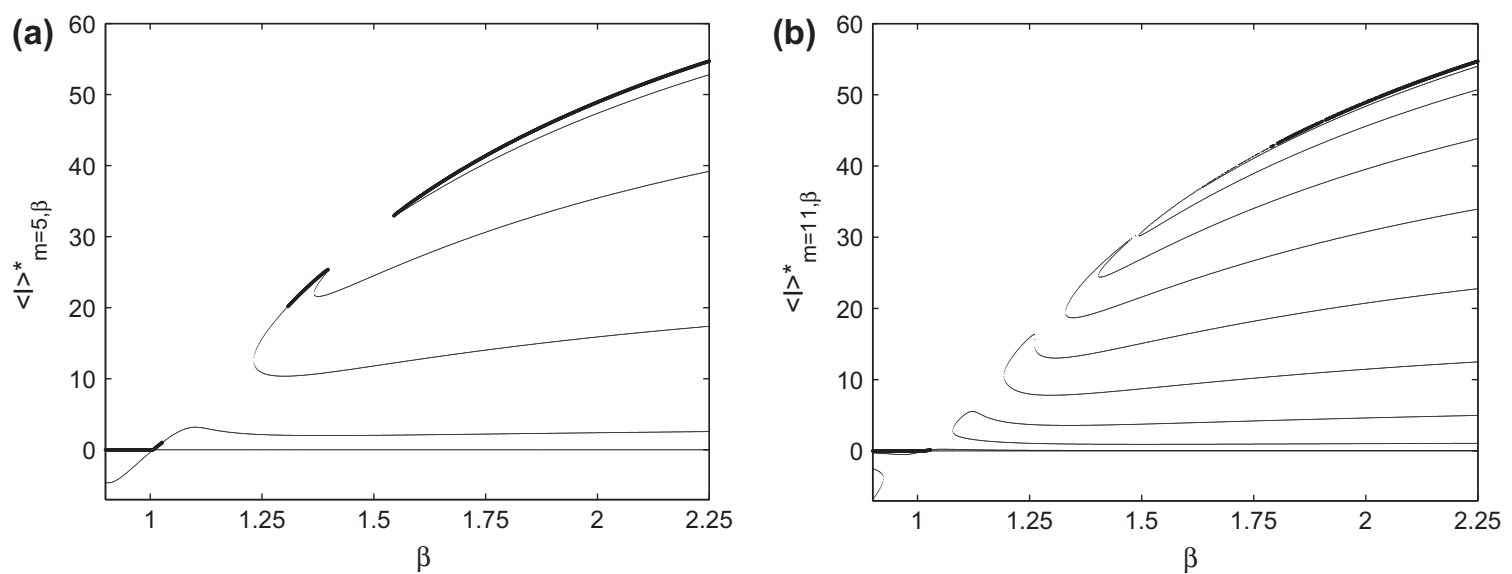

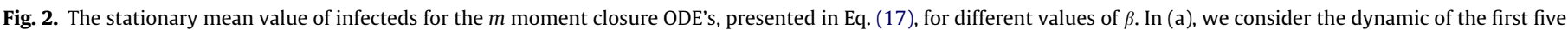

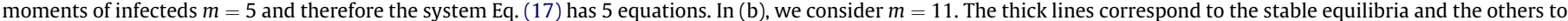
the unstable. The parameters $\alpha=1$ and $N=100$ were used

\subsection{Equilibria manifold}

To solve system Eq. (17) in stationarity for any dimension $m$ we use the following recursive process:

Step 1: Solve the first equation in the second moment

$$
\frac{d}{d t}\langle I\rangle=0 \Longleftrightarrow\left\langle I^{2}\right\rangle^{*}=\left(1-\frac{\alpha}{\beta}\right) N\langle I\rangle^{*}
$$

and substitute the result in the following equations;

Step 2: Solve the second equation in the third moment

$$
\frac{d}{d t}\left\langle I^{2}\right\rangle=0 \Longleftrightarrow\left\langle I^{3}\right\rangle^{*}=\left(\frac{\alpha}{\beta}+\left(1-\frac{\alpha}{\beta}\right)^{2} N\right) N\langle I\rangle^{*}
$$

and substitute the result in the following equations;

-..

Step $\mathbf{m}-\mathbf{1}$ :

Solve the $(m-1)$ th equation in the mth moment

$$
\frac{d}{d t}\left\langle I^{m-1}\right\rangle=0 \Longleftrightarrow\left\langle I^{m}\right\rangle^{*}=\left\langle I^{m}\right\rangle^{*}\left(\langle I\rangle^{*}\right)
$$

and substitute the result in the last equation.

In the end, we obtain a $(m+1)$ th order polynomial function in the first moment of infecteds

$\langle I\rangle^{*}\left(c_{m}\langle I\rangle^{* m}+\ldots+c_{1}\langle I\rangle^{*}+c_{0}\right)=0$,

that is numerically solved for fixed values of $\alpha, \beta$ and $N$. Furthermore, all the higher moments $\left\langle I^{n}\right\rangle^{*}, n \geqslant 2$, at equilibria are determined by the first moment of infecteds $\langle I\rangle^{*}$. We will denote by $\langle I\rangle_{m . \beta}^{*}$ the solutions of Eq. (18), i.e., the stationary mean value of infected individuals for the $m$ moment closure ODE system. In Fig. 2, we present the real zeros of the polynomial function given by Eq. (18) for $m=5$ and $m=11$ and different infection rate values $\beta$. The values used for the parameters $\alpha$ and $N$ are $\alpha=1$ and $N=100$. There are multiple equilibria and we present in thick lines the stable equilibria and in thin lines the unstable ones. We observe that the stable equilibrium is the equilibrium with the largest value of $\langle I\rangle^{*}$ because is the only one for which the jacobian matrix of system Eq. (17) has all eigenvalues with negative real part.

Let $\left[\beta_{m} ;+\infty\right)$ be the interval of the infection rate $\beta$ for which there is a stable equilibria for the $m$ moment closure ODE's. We observe that the value $\beta_{m}$ tends to $+\infty$ when $m$ tends to $+\infty$. Furthermore, letting the critical value of the infection rate $\beta_{c}(m)$ be the smallest value of $\beta$ such that the equilibrium $\langle I\rangle^{*}=0$ turns into an unstable equilibrium, for the $m$ moment closure ODE's, we also observe that $\beta_{c}(m)$ tends to $+\infty$ when $m$ tends to $+\infty$. These critical infection rate values $\beta_{c}(m)$ can be computed using the jacobian matrix of system Eq. (17) at the equilibrium $\langle I\rangle^{*}=\left\langle I^{2}\right\rangle^{*}=\ldots=\left\langle I^{m}\right\rangle^{*}=0$, that has a characteristic aspect given by

$$
J=\left[\begin{array}{cccccc}
\beta-\alpha & -\frac{\beta}{N} & 0 & 0 & 0 & \\
\beta+\alpha & 2(\beta-\alpha)-\frac{\beta}{N} & -2 \frac{\beta}{N} & 0 & 0 & \\
\beta-\alpha & 3(\beta+\alpha)-\frac{\beta}{N} & 3(\beta-\alpha)-3 \frac{\beta}{N} & -3 \frac{\beta}{N} & 0 & \\
\beta+\alpha & 4(\beta-\alpha)-\frac{\beta}{N} & 6(\beta+\alpha)-4 \frac{\beta}{N} & 4(\beta-\alpha)-6 \frac{\beta}{N} & -4 \frac{\beta}{N} & \\
& & & & \ddots
\end{array}\right] .
$$

\section{Results and discussion}

Nåsell [15] indicates that the stable equilibria of the 1 to 3 moment closure ODE's in cumulant truncation can be used to give a good approximation of the quasi-stationary mean value of infecteds $\langle I\rangle_{Q S, \beta}$ for high values of the population size $N$. Here, we study how this approximation can improve using higher moment closure ODE's. In Fig. 3, we present the distance $\left|\langle I\rangle_{m, \beta}^{*}-\langle I\rangle_{Q S, \beta}\right|$ between the first moment of infecteds obtained by the successive $m$ moment closure ODE's $\langle I\rangle_{m, \beta}^{*}$ and the quasi-stationary mean value of infecteds $\langle I\rangle_{O S, \beta}$ for the distribution presented in Eq. (7). In Fig. 3 a), we consider a relatively small infection rate $\beta=1.6$, above the critical mean field threshold $\beta_{c}=1$, taking $\alpha=1$ and $N=100$. We observe that the distance $\left|\langle I\rangle_{m, \beta=1.6}^{*}-\langle I\rangle_{Q S, \beta=1.6}\right|$ decreases with $m$ while the equilibria $\langle I\rangle_{m, \beta=1.6}^{*}$ are stable (up to $m=5$ ). In Fig. $3 \mathrm{~b}$ ), we consider other infection rate values and we observe that the distance $\left|\langle I\rangle_{m, \beta}^{*}-\langle I\rangle_{Q S, \beta}\right|$ decreases with $m$ up to a certain $m$ th moment closure approximation that can even occur before the break down of the stable equilibria for the value of the infection rate $\beta$ under consideration (see simulations for other transition rates in Pinto et al. $[18,19])$. Similar approximations are observed for the higher moments of infected individuals $\left\langle I^{n}\right\rangle^{*}, n \geqslant 2$.

\section{Conclusions}

Nåsell [15] studied the first 3 cumulants for the stochastic SIS model. Here, we improved the approximations by truncating at higher order cumulants. To accomplish this we developed a recursive method to compute the equilibria manifold of the $m$ moment 

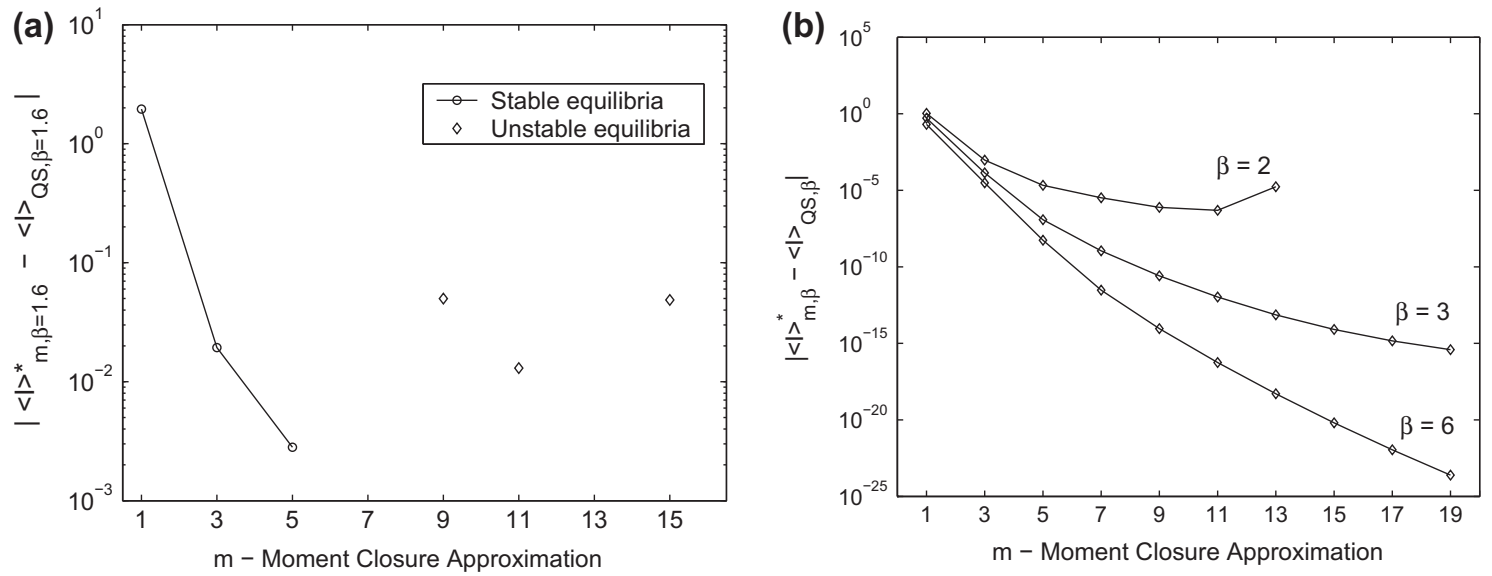

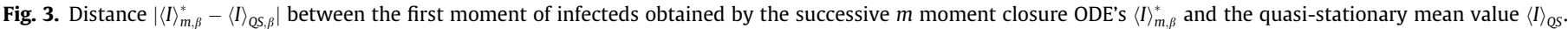

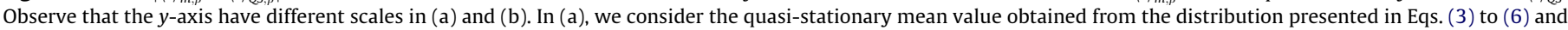
in (b), the approximated distribution presented in Eq. (7). The population size used is $N=100$.

closure ODE's, for every $m$. We showed that for high values of $m$ this method provides better approximations when the quasi-stationary distribution is highly skewed, which is more likely the case when either the population size is small or the infection rate is small or both.

\section{Acknowledgments}

The authors are grateful to the anonymous referees for their valuable comments and suggestions. We thank LIAAD-INESC Porto LA, Calouste Gulbenkian Foundation, Programs FEDER, POCTI and POCI by FCT and Ministério da Ciência, Tecnologia e Ensino Superior and Centro de Matemática da Universidade do Minho and Centro de Matemática e Aplicacćooes Fundamentais da Universidade de Lisboa for their financial support. José Martins also acknowledge the financial support from the FCT Grant with reference SFRW/BD/37433/2007. This work has been further supported by the European Union under FP7 in the EPIWORK project and the Portuguese FCT project PTDC/MAT/115168/2009.

\section{Appendix A. The moment closure function}

Proof (Proof of Lemma 1). We know that if $I$ is a random variable with the $n$ first moments $\left\langle I^{k}\right\rangle, k \in\{0,1, \ldots, n\}$, then it has cumulants of the same order that can be computed by the recursive formula given in Eq. (14) (see Smith [22], Papoulis [16]). Hence, assuming that $\left\langle\left\langle I^{m+1}\right\rangle\right\rangle=0$ we obtain that

$$
\begin{aligned}
\left\langle I^{m+1}\right\rangle & =\sum_{j=1}^{m}\left({ }^{m}{ }_{j}\right)\left\langle I^{j}\right\rangle\left\langle\left\langle I^{m+1-j}\right\rangle\right\rangle \\
& =m\langle I\rangle\left\langle\left\langle I^{m}\right\rangle\right\rangle+\sum_{j=2}^{m-1}\left({ }^{m}{ }_{j}\right)\left\langle I^{j}\right\rangle\left\langle\left\langle I^{m+1-j}\right\rangle\right\rangle+\left\langle I^{m}\right\rangle\langle\langle I\rangle\rangle .
\end{aligned}
$$

Applying the recursive formula given in Eq. (14) to compute the cumulant $\left\langle\left\langle I^{m}\right\rangle\right\rangle$, it follows that

$$
\begin{aligned}
\left\langle I^{m+1}\right\rangle= & m\langle I\rangle\left(\left\langle I^{m}\right\rangle-\sum_{j=1}^{m-1}\left({ }^{m-1}{ }_{j}\right)\left\langle I^{j}\right\rangle\left\langle\left\langle I^{m-j}\right\rangle\right\rangle\right) \\
& +\sum_{j=2}^{m-1}\left({ }^{m}{ }_{j}\right)\left\langle I^{j}\right\rangle\left\langle\left\langle I^{m+1-j}\right\rangle\right\rangle+\left\langle I^{m}\right\rangle\langle\langle I\rangle\rangle .
\end{aligned}
$$

We observe that only the first and the last terms of the previous expression depends on the mth moment. Hence, Eq. (A.2) can be reorganized in order to join the terms with this moment and we obtain

$$
\begin{aligned}
\left\langle I^{m+1}\right\rangle= & -m\langle I\rangle \sum_{j=1}^{m-1}\left({ }^{m-1}{ }_{j}\right)\left\langle I^{j}\right\rangle\left\langle\left\langle I^{m-j}\right\rangle\right\rangle+\sum_{j=2}^{m-1}\left({ }^{m}{ }_{j}\right)\left\langle I^{j}\right\rangle\left\langle\left\langle I^{m+1-j}\right\rangle\right\rangle \\
& +m\langle I\rangle\left\langle I^{m}\right\rangle+\left\langle I^{m}\right\rangle\langle\langle I\rangle\rangle .
\end{aligned}
$$

Defining the function $g_{m}$ by

$$
\begin{aligned}
g_{m}\left(\langle I\rangle,\left\langle I^{2}\right\rangle, \ldots,\left\langle I^{m-1}\right\rangle\right)= & -m\langle I\rangle \sum_{j=1}^{m-1}\left({ }^{m-1}{ }_{j}\right)\left\langle I^{j}\right\rangle\left\langle\left\langle I^{m-j}\right\rangle\right\rangle \\
& +\sum_{j=2}^{m-1}\left({ }^{m}{ }_{j}\right)\left\langle I^{j}\right\rangle\left\langle\left\langle I^{m+1-j}\right\rangle\right\rangle,
\end{aligned}
$$

we obtain for the moment $\left\langle I^{m+1}\right\rangle$ the equality

$\left\langle I^{m+1}\right\rangle=g_{m}\left(\langle I\rangle,\left\langle I^{2}\right\rangle, \ldots,\left\langle I^{m-1}\right\rangle\right)+(m+1)\langle I\rangle\left\langle I^{m}\right\rangle$,

as presented in Lemma 1.

\section{References}

[1] N.T.J. Bailey, The Mathematical Theory of Infectious Diseases and its Applications, second ed., Oxford University Press, New York, 1975.

[2] N.T.J. Bailey, The Elements of Stochastic Processes with Applications to the Natural Sciences, John Wiley \& Sons Inc., New York, 1964.

[3] F. Brauer, C. Castillo-Chavez, Mathematical Models in Population Biology and Epidemiology, Springer-Verlag, New York, 2001.

[4] F. Brauer, P. van den Driessche, J. Wu, Mathematical Epidemiology, SpringerVerlag, Berlin Heidelberg, Berlin, 2008.

[5] D. Clancy, S.T. Mendy, Approximating the quasi-stationary distribution of the SIS model for endemic infection, Method. Comput. Appl. Probab. 13 (3) (2011) 603-618.

[6] H.W. Hethcote, The basic epidemiology models: models, expressions for R0 parameter estimation, and applications, in: S Ma, Y. Xia (Eds.), Mathematical Understanding of Infectious Disease Dynamics, World Scientific Publishing Co. Pte. Ltd., 2008, pp. 1-61.

[7] R. Kryscio, C. Lefevre, On the extinction of the S-I-S stochastic logistic epidemic J. Appl. Prob. 26 (4) (1989) 685-694.

[8] I. Krishnarajah, A. Cook, G. Marion, G. Gibson, Novel moment closure approximations in stochastic epidemics, Bull. Math. Biol. 67 (2005) 855-873.

[9] E. Lukacs, Characteristic Functions, second ed., Griffin, London, 1970.

[10] T. Matis, I.G. Guardiola, Achieving moment closure through cumulant neglect, The Mathematica Journal, 2010, doi:dx.doi.org/10.3888/tmj.12-2.

[11] J.H. Matis, T.R. Kiffe, On approximating the moments of the equilibrium distribution of a stochastic logistic model, Biometrics 52 (1996) 980-991.

[12] I. Nåsell, The quasi-stationary distribution of the closed endemic SIS model, Adv. Appl. Prob. 28 (3) (1996) 895-932.

[13] I. Nåsell, On the quasi-stationary distribution of the stochastic logistic epidemic, Math. Biosci. 156 (1-2) (1999) 21-40. 
[14] I. Nåsell, Extinction and quasi-stationarity in the Verhulst logistic model, J. Theor. Biol. 211 (1) (2001) 11-27.

[15] I. Nåsell, Moment closure and the stochastic logistic model, Theor. Popul. Biol. 63 (2) (2003) 159-168.

[16] A. Papoulis, Probability, Random Variables, and Stochastic Processes, McGrawHill, Boston, 2002.

[17] P. Picard, Sur les modèles stochastique logistiques en démographie, Ann. Inst. Henri Poincaré B II (1965) 151-172.

[18] A. Pinto, J. Martins, N. Stollenwerk, The higher moments dynamic on SIS model, in: Theodore E. Simos et al. (Eds.), Numerical Analysis and Applied Mathematics, AIP Conference Proceedings, vol. 1168, 2009, pp. 1527-1530.

[19] A. Pinto, M. Aguiar, J. Martins, N. Stollenwerk, Dynamics of epidemiologica models, Acta Biotheor. 58 (4) (2010) 381-389.
[20] E. Renshaw, Modelling Biological Populations in Space and Time, Cambridge University Press, Cambridge, 1991.

[21] A. Singh, J.P. Hespanha, Moment Closure Techniques for Stochastic Models in Population Biology, in: Proceedings of the 2006 American Control Conference, Minneapolis, Minnesota, USA, 2006. Amer. Stat. 49 (2) (1995) 217-218.

[22] P.J. Smith, A recursive formulation of the old problem of obtaining moments from cumulants and vice versa, Amer. Stat. 49 (2) (1995) 217-218.

[23] N.G. van Kampen, Stochastic Processes in Physics and Chemistry, NorthHolland, Amsterdam, 1981.

[24] G.H. Weiss, M. Dishon, On the asymptotic behavior of the stochastic and deterministic models of an epidemic, Math. Biosci. 11 (3-4) (1971) 261-265.

[25] P. Whittle, On the use of the normal approximation in the treatment of stochastic processes, J. Roy. Statist. Soc. Ser. B 19 (2) (1957) 268-281. 